\title{
Total Body Water Distribution in Breast Cancer Survivors Following Cancer Rehabilitation
}

\author{
Laura Stefani ${ }^{1, *}$, Davide Palmerini ${ }^{1}$, Michele Corezzi ${ }^{1}$, Gabriele Mascherini ${ }^{1}$, Cristian Petri ${ }^{1}$, \\ Riggs J. Klika ${ }^{2}$ and Giorgio Galanti ${ }^{1}$ \\ 1 Sports and Exercise Medicine Center, University of Florence, 50121 Firenze, Italy; palme8@hotmail.it (D.P.); \\ michele.corezzi@stud.unifi.it (M.C.); gabriele.mascherini@unifi.it (G.M.); cristian.petri25@gmail.com (C.P.); \\ giorgio.galanti@unifi.it (G.G.) \\ 2 Department of Sports Medicine, Pepperdine University, Malibu, CA 90263, USA; \\ Riggs.Klika@pepperdine.edu \\ * Correspondence: laura.stefani@unifi.it; Tel.: +39-347-768-9030
}

Academic Editor: Giuseppe Musumeci

Received: 24 February 2017; Accepted: 17 April 2017; Published: 19 April 2017

\begin{abstract}
Cancer in humans is frequently associated with compartmentalization of body fluids as a result of sedentary behavior and pharmacological cellular toxicity. Total Body Water (TBW) in the general population is approximately $55-60 \%$ of body weight in adult males and $50-55 \%$ in adult females, while varying significantly in pathological conditions. Exercise is largely recognized as an important tool to TBW distribution. The purpose of this study was to investigate, for a least 12 months, the impact of physical activity on body water distribution in a sample of cancer patients and compare their responses to a sample of healthy controls. Cancer patients included 28 clinically stable female cancer patients diagnosed with breast cancer (aged $59 \pm 9$ years, weight $70.2 \pm 9.9 \mathrm{~kg}$, and Body Mass Index (BMI $26.7 \pm 5.4 \mathrm{~kg} \cdot \mathrm{m}^{2}$ ), who were enrolled in a year-long physical activity prescription program. The results indicated the absence of significant variations of TBW\% between the cancer patients and controls, however, there was a significant improvement in intracellular water content (ICW\%) at 6 months (T0: $51.1 \pm 3.9$ vs. T6: $52.4 \pm 4.1 ; p<0.05$ ) and at T12 (T0: $51.1 \pm 3.9$ vs. $\mathrm{T} 12: 53.6 \pm 3.1 ; p<0.005)$. In conclusion, in this small sample of cancer survivors, an unsupervised cancer rehabilitation program reduced the trend towards increased peripheral edema.
\end{abstract}

Keywords: hydration; cancer; physical activity

\section{Introduction}

Hydration status is an important clinical consideration in the long-term management of several chronic diseases and is an important factor controlling cellular protein turnover that may affect oncotic pressure [1]. Changes in hydrostatic pressure, oncotic pressure, and vascular permeability may result in abnormal fluid shifts in the body, resulting in altered intra-(ICW) vs. extracellular water (ECW) concentrations. Increased ECW results in soft tissue swelling due to expansion of the interstitial volume and is termed edema. Cancer related edema may be a result of altered oncotic pressure or lymphatic system obstruction. Additionally, cancer treatments often result in reduced patient physical activity, and increased sedentary behavior and any associated obesity are likely contributors to altered total body water distribution [2]. In addition, cancer patients with co-morbidities such as hypertension or diabetes [3] can further contribute to abnormal water distribution, which may lead to further increased risk of metabolic disease.

Total body water (TBW) (typically 55-60\% in men and 50-55\% in women) is important in overall health, but maintaining a ICW:ECW ratio of approximately 3:2, should be the goal in cancer patients. It is clear that severe dehydration and associated changes in ICW:ECW ratios (water loss of $>2 \%$ of body 
weight) leads to reduced overall physiologic and cognitive function, while mild dehydration (water loss $<1.5-2 \%$ of body weight), often seen in the general population, can increase the risk of edema.

Exercise is largely recognized as an important tool to regulate TBW distribution [4]. It is clear that a combination of aerobic and resistance training improves global fitness, cardiovascular performance, and quality of life of cancer patients [5]. It has been recently reported that unsupervised physical activity at moderate intensity aimed to maintain cardiovascular fitness [6] works well in the cancer survivor population. However, relatively few data are available about the impact of unsupervised physical activity programs on TBW distribution in cancer survivors. Therefore, the purpose of this study is to investigate TBW distribution in a group of breast cancer survivors participating in a cancer rehabilitation program.

\section{Materials and Methods}

\subsection{Population Studied}

Subjects for this study were recruited from a larger cohort of 145 cancer patients and included a subgroup composed of 28 female subjects with an average age $59 \pm 9$ years who were previously diagnosed with cancer and enrolled in our rehabilitation program from 2013 to 2016. Female breast cancer patients received one line of chemotherapy, in adjuvant or in palliative setting, and fourteen received a cardiotoxic chemotherapy agent (either anthracyclines or trastuzumab). There were 20 cases of quadrantectomy and eight mastectomies performed on the female breast cancer patients. Additionally, the breast cancer (BC) patients underwent conventional adjuvant chemotherapy including: doxorubicin and cyclophosphamide or cyclophosphamide, methotrexate and 5-fluorouracile, or 5-fluorouracile, epirubicin, and cyclophosphamide for approximately three cycles, where one cycle is defined as a-course of therapy in a monthly interval. Ten subjects were prescribed hormone therapy (aromatase inhibitors) and all BC subjects had radiotherapy.

All subjects were evaluated by an oncologist and then referred to the Sports Medicine Center of the same university, where further evaluation and screening were completed. Secondary medical evaluation revealed the absence of any co-morbidities (cardiovascular disease, diabetes mellitus, metabolic syndrome).

At the initial phase of the study (T0), all subjects were in stable clinical condition, without evidence of metastatic disease or clinical and instrumental signs of cardiotoxicity. All cancer subjects were post-chemotherapy treatment for approximately 1-2 years before entry into the program. The cancer group was matched to healthy subjects free of cardiovascular risk factors ( $n=23$ subjects; male, $n=10(43 \%)$ and female, $n=13(57 \%)$, average age $53 \pm 13$ years), who followed the same physical activity program. Assessments on all subjects were completed at 0, 6, and 12 months (T0, T6, T12, respectively). The local ethics committee approved the study and all the patients gave their written consent to participate in the study.

\subsection{Protocol Study}

All subjects completed a questionnaire [7] to estimate their daily level of physical activity for further classification: sedentary or moderately active. The intensity of the daily activities was estimated in terms of metabolic equivalents (METS) and compared to standard Physical Activity Levels (PALs) [8]. A PAL level of 1.7 METS. day $^{-1}$ was used as the criteria to categorize sedentary versus active subjects [9]. A periodic supervision of physical activity was possible by the use of recurrent mobile phone calls during which adherence to the physical activity was monitored by the using of simple questions.

At the first examination (T0), at six months (T6), and at the end of the protocol (T12), stature, bodyweight, body mass index (BMI), waist and hip circumferences, and hydration status were determined. Hydration status, including TBW and distribution, was estimated using bioelectrical impedance analysis (BIA-101, Akern-RJL Systems, Florence, Italy). The measurements were made under standard conditions at the temperature of $20-24{ }^{\circ} \mathrm{C}$, with the patient supine on a plane 
nonconductive surface keeping upper limbs abducted at $30^{\circ}$ and lower limbs at $45^{\circ}$, as per the manufacturer's instructions.

Each subject then completed a graded exercise test to volitional exhaustion with echocardiography monitoring. Standard 2D echocardiography (My Lab 50 echocardiograph Esaote-Italy equipped with a $2.5 \mathrm{MHz}$ probe) was performed and hemodynamic response to exercise was recorded (systolic and diastolic blood pressure). This non-invasive cardiological evaluation, as echo fast exam, was done to exclude those subjects with signs of potential heart failure. For this reason, only the 2D echo standard measures of the systolic and diastolic parameters were performed, with particular attention to the Ejection Fraction and to exclude the eventual valves dysfunction. No substantial morphological modifications of the heart's function were found and for this reason, the echocardiographic data are not reported. Peak heart rate (HRpeak), blood pressure, and rating of perceived exertion (RPE) was recorded and used to determine physical activity intensity in the rehabilitation program.

Each subject also performed a six-minute walk test (6 MWT) which was used in subsequent follow-up testing [10] to evaluate cardiorespiratory improvement. The 6 MWT is both reliable and valid in the evaluation of chronically diseased patients, especially when paired with the Borg or CR-10 rating of perceived exertion scale [11].

Resistance training exercises were determined based on the results of hand-grip dynamometry and chair stand tests. In the hand-grip test, the subject holds a dynamometer in the hand to be tested, with the arm at a right angle and the elbow by the side of the body. When ready, the subject squeezes the dynamometer with maximum isometric effort, which is maintained for five seconds. The subject is verbally encouraged to give a maximum effort and the effort is recorded in kilograms.

During the chair stand test, the subject sits in the middle of a stabilized chair, with their feet shoulder width apart, flat on the floor. The arms are crossed at the wrists and held close to the chest. From the sitting position, the subject stands completely up, then completely back down for $30 \mathrm{~s}$. The total number of complete chair stands is recorded.

Exercise interventions were based on the American College of Sports Medicine (ACSM) exercise guidelines for cancer survivors [8] and individually tailored to each subject based on their graded exercise tests and strength assessments. Aerobic exercise intensity was prescribed at $60 \%$ of peak heart rate determined from the maximal treadmill test and consisted primarily of walking. Resistance training consisted of eight exercises targeting major muscle groups, with three sets and 10-15 repetitions, mainly with free weight or using body weight.

The program was 12 months long and consisted of individual physical activity sessions being completed, unsupervised at home. Subjects were monitored via mobile phone weekly where either physical activity (completed or not) or nutrition habits were recorded. Follow-up phone contact has been shown to increase adherence to home-based physical activity programs [12].

All the subjects were instructed to follow a typical Mediterranean diet throughout the study period, where the whole daily calories were divided in five meals, three main meals and two snacks.

The diet was generally determined by looking at a pre-existing model, where the caloric intake was composed of: $50-60 \%$ carbohydrates, not more than $20 \%$ coming from simple sugars; a large portion of vegetable fibers, always below than $35 \mathrm{~g}$ /day; between $12 \%$ and $18 \%$ of vegetable and animal proteins; and the remaining $25-30 \%$ was provided by lipids, with just $25 \%$ of these lipids composed of saturated fatty acids.

Each main meal should have included 1-2 portions of vegetables, fruits, and cereals, with whole cereals preferred for grains.

Daily consumption should also have included 1-2 portions of milk and dairy products, preferably low-fat; 3-4 portions of extra virgin olive oil, and 1-2 portions of dried fruit and seeds.

The weekly consumption of proteins should have been made up of: white meat 2-3 times per week with just one portion of red meat per week, fish at least 2 times per week, preferably bluefish, eggs 2-3 times per week, and legumes at least 2 times per week. 
Participants were also reminded of the importance of good hydration, with the suggestion that they should consume 1.5-2 L of water every day, far from meals, and preferably in small amounts.

Participants were recommended not to add too much salt to their meals, to always employ simple ways of cooking, and to avoid using butter or oil for cooking.

\subsection{Bioelectrical Analysis}

Unlike other techniques used to assess body composition, such as underwater weighing (densitometry), dual energy X-ray absorptiometry (DEXA), and magnetic resonance imaging (MRI) which are expensive, inconvenient for the participant, and not feasible to conduct in the field because they require large specialized equipment, bioelectrical impedance analysis (BIA) is by contrast relatively simple, quick, and non-invasive. The results are available immediately and reproducible [13].

BIA was used to assess hydration status and distribution of body water and was carried out according to the recommendations of the National Institute of Health( NIH) Consensus Statement.

The measurements were done on the right side of the body according to the manufacturer's instructions [14].

In addition to (TBW) and distribution (ICW and ECW), other measures included body cellular mass (BCM), extra cellular mass (ECM), and the phase angle (PA). BCM represents the total mass of all cellular elements where main metabolic processes occur. In the human body, the phase angle is used to determine intra- and extracellular quantities, which represent an important prognostic index used to monitor the presence and evolution of chronic inflammatory processes. The use of BIA in this setting is non-invasive, cost-effective, and provides reliable data on total body water.

\subsection{Statistical Analysis}

Descriptive statistics were calculated on all variables and presented as mean \pm standard deviation. A repeated measures analysis of variance (ANOVA) was used to test means across time. The level of significance was set at $p<0.05$ and comparisons between $\mathrm{T} 0$ and $\mathrm{T} 6$ and between $\mathrm{T} 6$ and $\mathrm{T} 12$ were made in order to give more information regarding modification trends. The data were analyzed using the statistical package IBM SPSS Statistics (version 13.0) for Windows. (SPSS Inc., Chicago, IL, USA).

\section{Results}

All subjects completed the 12-month study protocol and no adverse events were recorded. Body weight and BMI were unchanged over the course of the study, however, there were significant changes in body water distribution.

A significant increase of ICW\% at T6 and T12 which is related to a significant reduction of ECW\% at $\mathrm{T} 6$ and at $\mathrm{T} 12$ was observed, despite the absence of a significant variation of TBW\% (see Table 1 and Figures 1 and 2). Additionally, there were significant increases in PA and BCM at 6 and 12 months of physical activity with concomitant decreases in ECM (Figures 3-5).

Table 1. Comparison of selected variables after 6 and 12 months of cancer rehabilitation.

\begin{tabular}{ccccccc}
\hline $\boldsymbol{n}=\mathbf{2 8}$ Cancer Patients & T0 & T6 & P $\mathbf{\Delta t 0 - t 6}$ & T12 & P $\mathbf{\Delta t 0 - t 1 2}$ & ANOVA Test \\
\hline Weight $(\mathrm{kg})$ & $70.2 \pm 9.9$ & $69.9 \pm 14.9$ & NS & $70.5 \pm 15.8$ & NS & NS \\
BMI $\left(\mathrm{kg} \cdot \mathrm{m}^{-2}\right)$ & $26.7 \pm 5.4$ & $26.6 \pm 5.6$ & NS & $26.8 \pm 5.8$ & NS & NS \\
PA ( $\left.{ }^{\circ}\right)$ & $5.4 \pm 0.7$ & $5.7 \pm 0.8$ & $<0.05$ & $5.9 \pm 0.7$ & $<0.005$ & $<0.05$ \\
FM (\%) & $34.6 \pm 8.3$ & $34.4 \pm 8.5$ & NS & $33.7 \pm 10.3$ & NS & NS \\
FFM (\%) & $65.4 \pm 8.3$ & $65.6 \pm 8.5$ & NS & $66.3 \pm 10.3$ & NS & NS \\
TBW (\%) & $49.2 \pm 5.6$ & $49.5 \pm 6.0$ & NS & $50.0 \pm 7.0$ & NS & NS \\
ECW (\%) & $48.9 \pm 3.9$ & $47.6 \pm 4.1$ & $<0.05$ & $46.4 \pm 3.1$ & $<0.005$ & $<0.05$ \\
ICW (\%) & $51.1 \pm 3.9$ & $52.4 \pm 4.1$ & $<0.05$ & $53.6 \pm 3.1$ & $<0.005$ & $<0.05$ \\
BCM (\%) & $50.3 \pm 4.2$ & $51.7 \pm 4.4$ & $<0.05$ & $53.0 \pm 3.4$ & $<0.005$ & $<0.05$ \\
ECM $(\%)$ & $49.7 \pm 4.2$ & $48.3 \pm 4.4$ & $<0.05$ & $47.0 \pm 3.4$ & $<0.005$ & $<0.05$ \\
\hline
\end{tabular}

Legend: (BMI) Body Mass Index; (PA) Phase Angle; (FM) Fat Mass; (FFM) Free Fat Mass; (TBW) Total Body Water; (ECW) Extra Cellular Water; (ICW) Intra Cellular Water; (BCM) Body Cellular Mass; (ECM) Extra Cellular Mass. 


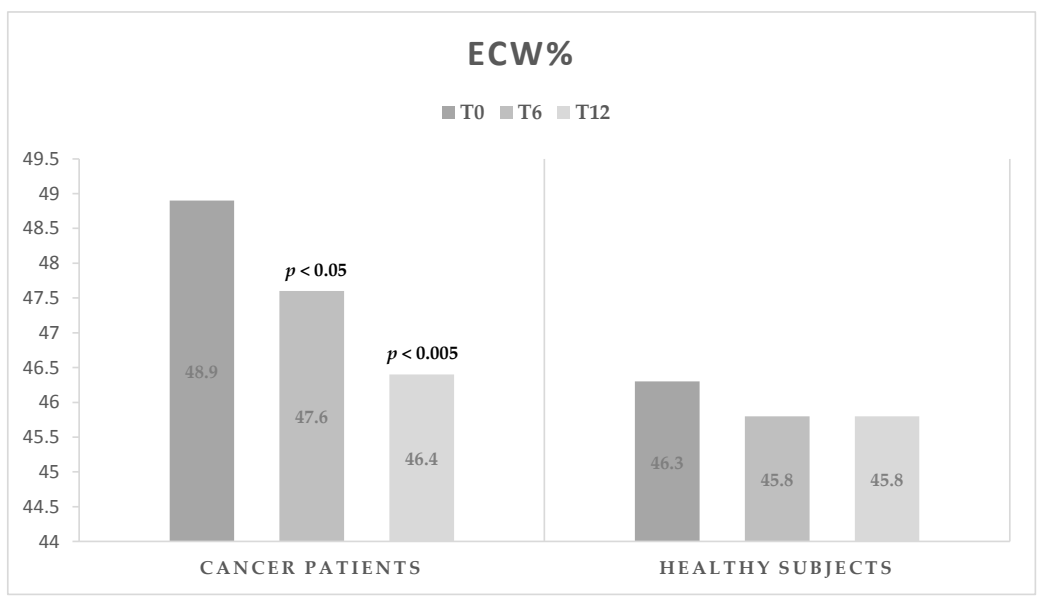

Figure 1. Extra cellular water (ECW) percentage at T0, T6, and T12. Significant decreases in ECW\% were observed at T6 and T12 in cancer patients; no significant variation in the healthy subjects group was observed.

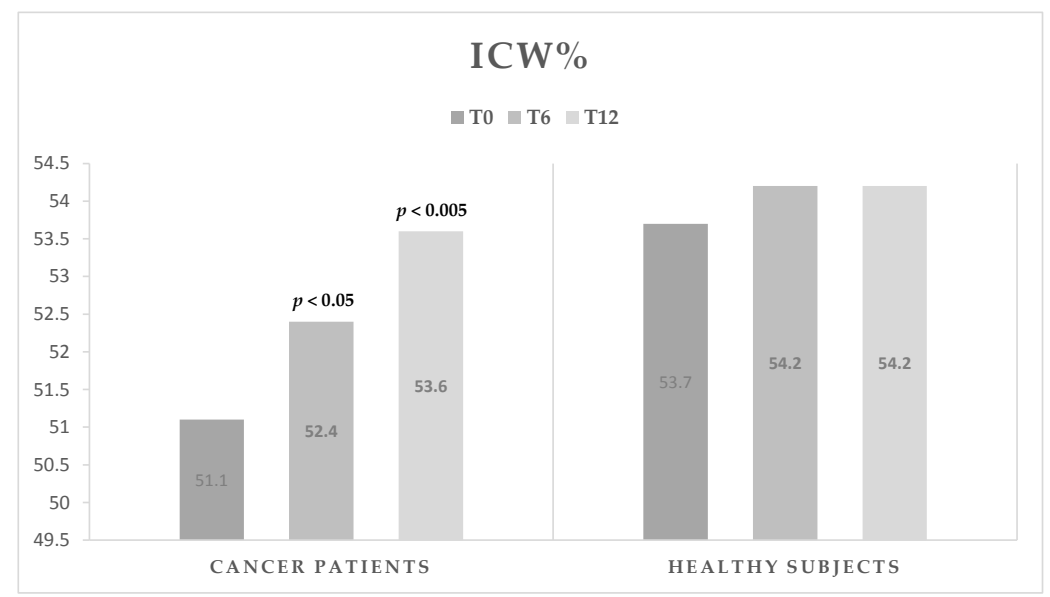

Figure 2. Intracellular water (ICW) percentage at T0, T6, and T12. Significant increases in ICW\% were observed at T6 and T12 in cancer patients; no significant variation in the healthy subjects group was observed.

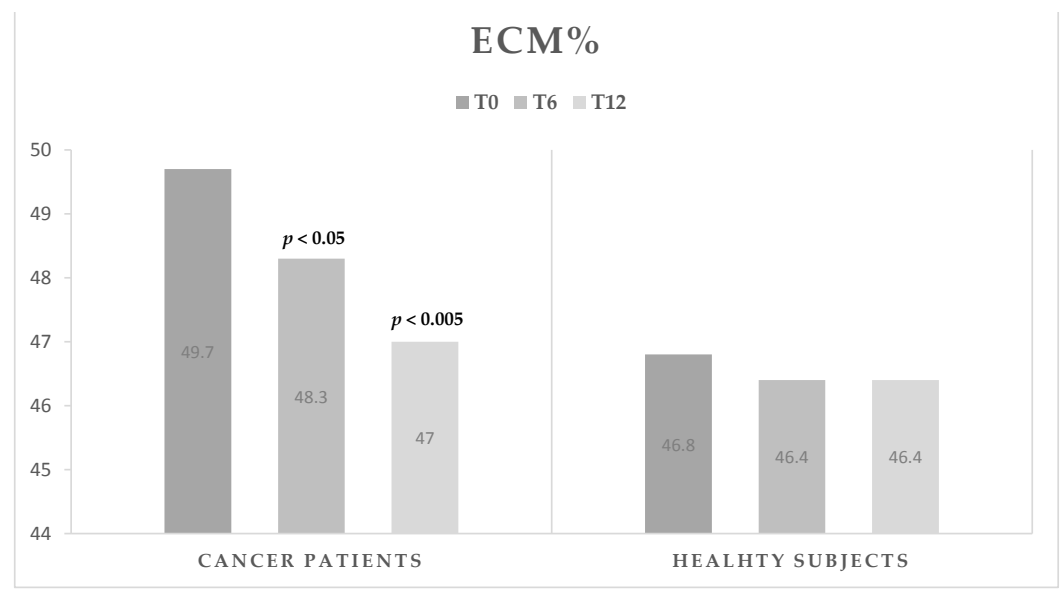

Figure 3. Extracellular water mass (ECM) percentage at T0, T6, and T12. Significant decreases in ECM\% were observed at T6 and T12 in cancer patients; no significant variation in the healthy subjects group was observed. 


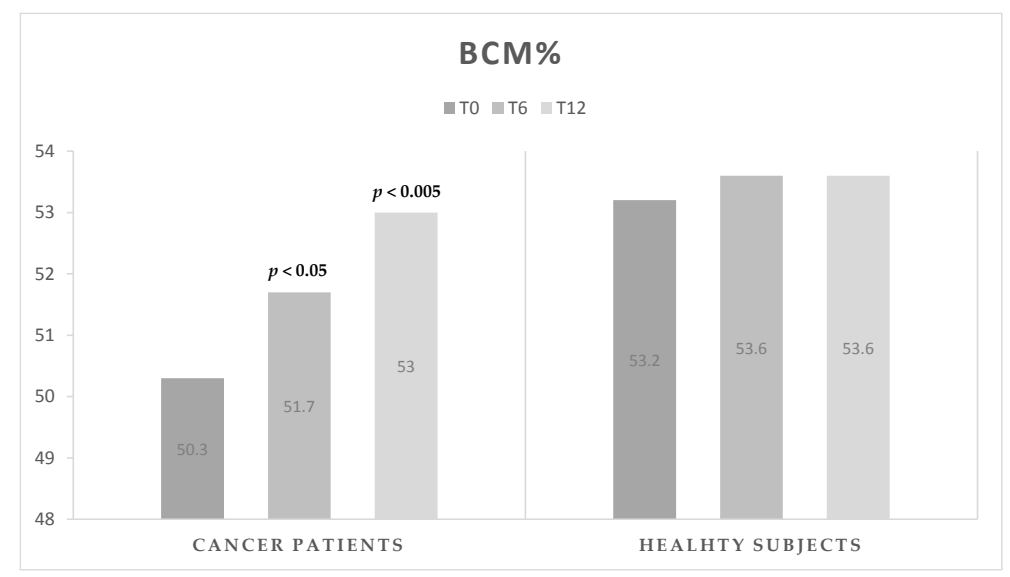

Figure 4. Body cellular mass (BCM) percentage at T0, T6, and T12. Significant increases in BCM\% were observed at T6 and T12 in cancer patients; no significant variation in the healthy subjects group was observed.

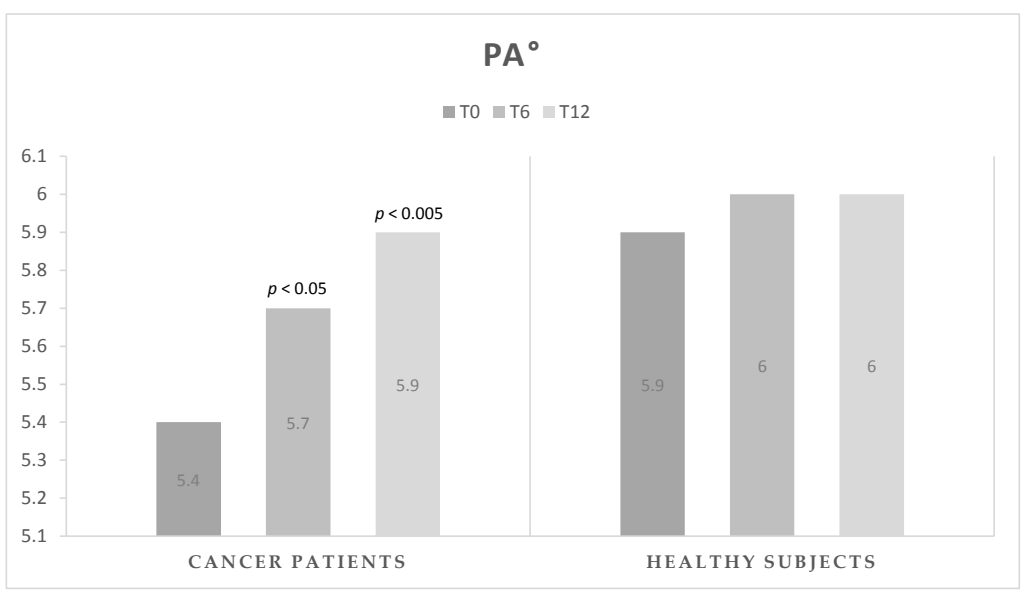

Figure 5. Phase angle (PA) at T0, T6, and T12. Significant increases in PA at T6 and T12 were observed in cancer patients; no significant variation in the healthy subjects group was observed.

In the group of active healthy subjects, there were no significant changes observed in the follow-up at 6 and 12 months in regard to the intra and extra cellular hydration, while there was a significant increase in TBW\% at 6 months (see Table 2).

Table 2. Comparison of selected variables after 6 and 12 months of physical activity program.

\begin{tabular}{ccccccc}
\hline $\boldsymbol{n}$ = 23 Healthy Subjects & T0 & T6 & P $\mathbf{\Delta t 0 - t 6}$ & T12 & P $\mathbf{\Delta t 0 - t 1 2}$ & ANOVA Test \\
\hline Weight $(\mathrm{kg})$ & $76.6 \pm 12.3$ & $76.0 \pm 11.6$ & NS & $75.6 \pm 11.4$ & NS & NS \\
BMI & $26.1 \pm 2.8$ & $25.8 \pm 2.4$ & NS & $25.6 \pm 2.5$ & NS & NS \\
PA ( $\left.{ }^{\circ}\right)$ & $5.9 \pm 0.9$ & $6.0 \pm 0.9$ & NS & $6.0 \pm 0.9$ & NS & NS \\
FM (\%) & $28.8 \pm 5.4$ & $27.5 \pm 6.4$ & $<0.05$ & $28.0 \pm 5.4$ & NS & NS \\
FFM (\%) & $71.2 \pm 5.4$ & $72.5 \pm 6.4$ & $<0.05$ & $72.0 \pm 5.4$ & NS & NS \\
TBW (\%) & $53.0 \pm 4.4$ & $54.1 \pm 5.0$ & $<0.05$ & $53.7 \pm 4.3$ & NS & NS \\
ECW (\%) & $46.3 \pm 4.2$ & $45.8 \pm 3.9$ & NS & $45.8 \pm 4.6$ & NS & NS \\
ICW (\%) & $53.7 \pm 4.2$ & $54.2 \pm 3.9$ & NS & $54.2 \pm 4.6$ & NS & NS \\
BCM (\%) & $53.2 \pm 4.5$ & $53.6 \pm 4.3$ & NS & $53.6 \pm 5.0$ & NS & NS \\
ECM $(\%)$ & $46.8 \pm 4.5$ & $46.4 \pm 4.3$ & NS & $46.4 \pm 5.0$ & NS & NS \\
\hline
\end{tabular}

Legend: (BMI) Body Mass Index; (PA) Phase Angle; (FM) Fat Mass; (FFM) Free Fat Mass; (TBW) Total Body Water; (ECW) Extra Cellular Water; (ICW) Intra Cellular Water; (BCM) Body Cellular Mass; (ECM) Extra Cellular Mass. 


\section{Discussion}

Many metabolic chronic diseases including cancer are associated with abnormal water distribution [14,15]. Proper hydration balance is an important medical consideration, as abnormal TBW status and distribution can increase the risk of cardiovascular pathologies including thrombogenesis. Additionally, it has also been shown that impaired hydration status can have a negative impact on overall survival, even in healthy people $[16,17]$. Therefore, it is critical to monitor and adjust abnormalities in TBW and water distribution. It was the goal of this study to see if TBW and distribution could be altered with a home-based rehabilitation program consisting of unsupervised aerobic and resistance training in a breast cancer group.

In this study, regular moderate physical activity, which is typically prescribed to increase cardiorespiratory fitness and strength, was used as a stimulus to activate skeletal muscle, which assisted in a redistribution of TBW to a more clinically stable standard. This is an important component of the cancer rehabilitation program, as it may help increase skeletal muscle performance and indirectly decrease the risk of musculoskeletal damage [18].

Several methods have been used to assess and quantify hydration status. The non-invasive method of BIA used in this study proved to be a reliable and effective approach to monitor tissue body water in breast cancer patients and is similar to data presented elsewhere [19]. Additionally, the data from this study demonstrate BIA was sensitive enough to detect fluid shifts after a moderate physical activity program that was independent of body weight changes. The progressive fluid shift from intracellular to extracellular compartments seen by positive phase angle changes in this study is similar to the mechanism by which the exercise is used to reduce edema.

Lastly, the results of this study also provide supporting evidence that physical activity adherence can be high when using phone call-initiated follow ups and that a home-based rehabilitation program was effective in modifying tissue water distribution [20].

\section{Conclusions}

Hydration status, in the dynamic concept of body fluid distribution, is one of the principal components related to the survival of diseased patients. Given that hydration in advanced cancer is poorly understood, it is important to advance the assessment of hydration in this group. By using physical activity as a therapeutic post-cancer treatment, not only can functionality and quality of life be improved, but tissue water distribution can be restored, thereby further improving physiologic function and overall health.

Author Contributions: Laura Stefani conceived and wrote the manuscript. Davide Palmerini and Michele Corezzi elaborated the data and contributed to writing the manuscript. Gabriele Mascherini and Cristian Petri collected the data for all the observation periods. Riggs J. Klika revised the entire manuscript and improved the message of the manuscript. Giorgio Galanti revised and approved the final version of the manuscript.

Conflicts of Interest: The authors declare no conflict of interest.

\section{References}

1. Haussinger, D.; Roth, E.; Lang, F.; Gerok, W. Cellular hydration state: An important determinant of protein catabolism in health and disease. Lancet 1993, 341, 1330-1332. [CrossRef]

2. Marken Lichtenbelt, W.D.; Fogelholm, M. Increased extracellular water compartment, relative to intracellular water compartment, after weight reduction. J. Appl. Physiol. 1999, 87, 294-298. [PubMed]

3. Wareham, N.J.; van Sluijs, E.M.; Ekelund, U. Physical activity and obesity prevention: A review of the current evidence. Proc. Nutr. Soc. 2005, 64, 229-247. [CrossRef] [PubMed]

4. Manz, F.; Wentz, A. The importance of good hydration for the prevention of chronic disease. Nutr. Rev. 2005, 63, 2-5. [CrossRef]

5. Stefani, L.; Galanti, G.; Klika, R. Clinical implementation of exercise guidelines for cancer patients: Adaptation of ACSM's guidelines to the Italian model. J. Funct. Morphol. Kinesiol. 2017, 2, 4. [CrossRef] 
6. Garber, C.E.; Blissmer, B.; Deschenes, M.R.; Franklin, B.A.; Lamonte, M.J.; Lee, I.M.; Nieman, D.C.; Swain, D.P.; ACSM position stand. Quantity and quality of exercise for developing and maintaining cardiorespiratory, musculoskeletal, and neuromotor fitness in apparently healthy adults: Guidance for prescribing exercise. Med. Sci. Sports Exerc. 2011, 43, 1334-1359. [CrossRef] [PubMed]

7. McArdle, W.D.; Katch, F.I.; Katch, V.L. Exercise Physiology: Nutrition, Energy, and Human Performance; Lippincott Williams \& Wilkins: Philadelphia, PA, USA, 2015.

8. Pescatello, L.S.; Arena, R.; Riebe, D.; Thompson, P.D. ACSM's Guidelines for Exercise Testing and Prescription, 9th ed.; Wolters Kluwer/Lippincott Williams \& Wilkins: Philadelphia, PA, USA, 2014; p. 456.

9. Human Energy Requirements: Energy Requirement of Adults. Report of a Joint FAO/WHO/UNU Expert Consultation. Food and Agriculture Organization of the United Nations, 2004. Available online: http:/ /www.fao.org/docrep/007/y5686e/y5686e00.htm (accessed on 18 April 2017).

10. Guyatt, G.H.; Sullivan, M.J.; Thompson, P.J.; Fallen, E.L.; Pugsley, S.O.; Taylor, D.W.; Berman, L.B. The 6-minute walk: A new measure of exercise capacity in patients with chronic heart failure. Can. Med. Assoc. J. 1985, 132, 919-923. [PubMed]

11. Robertson, R.J.; Goss, F.L.; Dubé, J.; Rutkowski, J.; Dupain, M.; Brennan, C.; Andreacci, J. Validation of the adult OMNI scale of perceived exertion for cycle ergometer exercise. Med. Sci. Sports Exerc. 2004, 36, 102-108. [CrossRef] [PubMed]

12. WHO. Adherence to Long-Term Therapies: Evidence for Action; World Health Organization: Geneva, Switzerland, 2003.

13. Dehghan, M; Merchant, A. Is bioelectrical impedance accurate for use in large epidemiological studies? Nutr. J. 2008, 7, 26. [CrossRef] [PubMed]

14. Kyle, U.G.; Bosaeus, I.; de Lorenzo, A.D.; Deurenberg, P.; Elia, M.; Gómez, J.M.; Pichard, C. Bioelectrical impedance analysis-Part I: Review of principles and methods. Clin. Nutr. 2004, 23, 1226-1243. [CrossRef] [PubMed]

15. Waki, M.; Kral, J.G.; Mazariegos, M.; Wang, J.; Pierson, R.N.; Heymsfield, S.B. Relative expansion of extracellular fluid in obese vs. nonobese women. Am. J. Physiol. 1991, 261, 199-203.

16. Human Energy Requirements. Report of a Joint FAO/WHO/UNU Expert Consultation; FAO Food and Nutrition Technical Report Series 1; FAO/WHO/UNU: Rome, Italy, 2001.

17. Knudsen, N.N.; Kjærulff, T.M.; Ward, L.C.; Sæbye, D.; Holst, C.; Heitmann, B.L. Body water distribution and risk of cardiovascular morbidity and mortality in a healthy population: A prospective cohort study. PLoS ONE 2014, 3, e87466. [CrossRef] [PubMed]

18. Judelson, D.A.; Maresh, C.M.; Anderson, J.M.; Armstrong, L.E.; Casa, D.J.; Kraemer, W.J.; Volek, J.S. Hydration and muscular performance: Does fluid balance affect strength, power and high-intensity endurance? Sports Med. 2007, 37, 907-921. [CrossRef] [PubMed]

19. Gupta, D.; Lammersfeld, C.A.; Burrows, J.L.; Dahlk, S.L.; Vashi, P.G.; Grutsch, J.F.; Hoffman, S.; Lis, C.G. Bioelectrical impedance phase angle in clinical practice: Implications for prognosis in advanced colorectal cancer. Am. J. Clin. Nutr. 2004, 80, 1634-1638. [PubMed]

20. Stefani, L.; Petri, C.; Mascherini, G.; Galanti, G. Lifestyle intervention in surviving cancer patients. J. Funct. Morphol. Kinesiol. 2016, 1, 48-53. [CrossRef]

(C) 2017 by the authors. Licensee MDPI, Basel, Switzerland. This article is an open access article distributed under the terms and conditions of the Creative Commons Attribution (CC BY) license (http:/ / creativecommons.org/licenses/by/4.0/). 